\title{
APLICAÇÃO DA TOXINA BOTULÍNICA NA ODONTOLOGIA PARA CORREÇÃO DO SORRISO GENGIVAL
}

\section{APPLICATION OF BOTULINIC TOXIN FOR CORRECTION OF GUMMY SMILE}

\author{
Luiz Feliphe da Silva Bourscheid \\ Graduado em Odontologia pela Universidade de Rio Verde - UniRV, Brasil \\ E-mail: luizbourscheid@hotmail.com
}

Marlana Barbosa Carrijo de Carvalho Mestre em Periodontia, Universidade de Rio Verde - UniRV, Brasil E-mail: marlana@unirv.edu.br

Lorena Souza Santos Mariano Mestre em Endodontia, Universidade de Rio Verde - UniRV, Brasil

E-mail: lorena@unirv.edu.br

Kênia Alves Barcelos Doutoranda em Ciência Animal, Universidade de Rio Verde - UniRV, Brasil E-mail: keniabarcelos@unirv.edu.br

Lara Cândida de Sousa Machado Mestre em Ciências Ambientais e Saúde, Universidade de Rio Verde - UniRV, Brasil E-mail: laramachado.enf@gmail.com

Renato Canevari Dutra da Silva Doutorando em Saúde Coletiva, Universidade de Rio Verde - UniRV, Brasil

E-mail: renatocanevari@unirv.edu.br

Recebido: 08/06/2021 - Aceito: 20/06/2021

\section{RESUMO}

O sorriso gengival é uma das principais queixas dos pacientes, devido à sua influência na autoestima. A estrutura do sorriso é formada por: lábios, dentes e gengiva. A exibição gengival excessiva durante o sorriso é considerada indesejável e menos agradável. Sabe-se que possui causa multifatorial e pode estar presente no crescimento vertical excessivo da maxila, contração labial excessiva, lábio superior curto e extrusão dos dentes anteriores. Várias opções de tratamento estão disponíveis para a correção do sorriso gomoso. Recentemente, o uso de botox (toxina botulínica) tem sido preconizado no tratamento do sorriso gengival. A injeção minimamente invasiva de botox nos músculos hiperativos do lábio superior reduz o movimento ascendente do lábio, resultando em menos sorriso gengival. O uso da toxina botulínica pode estar associado a tratamentos adicionais ou ser aplicado individualmente, de acordo com a necessidade de cada paciente. Sendo usada corretamente, a toxina é 
uma opção de tratamento satisfatória e sua indicação é considerada um método de tratamento: rápido, eficaz, fácil e seguro, sendo uma abordagem mais conservadora quando comparada aos procedimentos cirúrgicos.

Palavras-chave: estética; bem-estar; toxinas botulínicas.

\begin{abstract}
Gummy smile is one of the major complaints of patients, due to its influence on the self-esteem. The framework of smile is formed by lips, teeth and gingival. Excessive gingival display during smile is considered undesirable and less pleasing. It is known that it has a multifactorial cause and can be present in the excessive vertical growth of the maxilla, excessive labial contraction, short upper lip and extrusion of the anterior teeth. Various treatment options are available for correction of gummy smile. Recently the use of boot (botulinum toxin) has been advocated in treatment of gummy smile. Minimally invasive injection of botox into the hyperactive muscles of upper lip reduces the upward movement of lip thus, resulting in less of gummy smile. The use of the botulinum toxin can be associated with additional treatments or be applied individually, according to the need of each patient. If used correct, the toxin is a satisfactory treatment option and its indication is considered a fast, effective, easy and safe treatment method, being a more conservative approach compared to surgical procedures.
\end{abstract}

Keywords: esthetics, welfare, botulinic toxin

\title{
1. INTRODUÇÃO
}

A estética facial é de grande importância para indivíduos que procuram tratamento médico cosmético. Tanto é, que várias pessoas procuram ajuda profissional para resolver problemas psicossociais causados, por uma aparência facial não estética. Dos problemas comumente relatados, o "sorriso gengival" é um deles (SEIXAS et al., 2011).

O sorriso estético é aquele que apresenta certa correlação harmônica entre forma e cor dos elementos dentários, e entre lábios e gengiva (MARSON, 2012; 2014). Quando o indivíduo apresenta mais de $3 \mathrm{~mm}$ de exposição gengival durante o sorriso, esse é denominado sorriso gengival (SENISE, 2015). Uma exibição gengival normal entre a borda inferior do lábio superior e a margem gengival dos incisivos centrais durante um sorriso normal é de 1 a $2 \mathrm{~mm}$. Por outro lado, se a distância é de $4 \mathrm{~mm}$ ou mais entre a gengiva e o lábio, é classificada como pouco atraente (VAN DER GELD 
et al., 2011).

Um sorriso gengival afeta o estado estético e psicológico dos pacientes, pois geralmente diminui a autoconfiança, levando a ocultar ou controlar o sorriso. A exposição gengival excessiva é uma causa comum dos pacientes que se queixam de sorriso gengival na rotina prática diária do consultório. Portanto, o clínico precisa avaliar o sorriso do paciente e também considerar a relação entre a dentição do paciente, gengiva e lábios enquanto sorri.

O sorriso gengival é uma das queixas mais comuns dos pacientes que procuram um sorriso estético. Existem várias alternativas para corrigir um sorriso gengival, variando desde técnicas como gengivectomia a procedimentos mais complexos e invasivos, como cirurgia ortognática. Desta forma, a escolha do tratamento conservador e minimamente invasivo com a utilização de toxina botulínica é recomendado por tratar-se de um método simples, rápido e eficaz. Evitando assim, métodos altamente complexos e caros, associados a demandas de longo prazo.

Em relação a outras técnicas, o uso de toxina botulínica consiste em uma técnica: simples, fácil e segura; durante a aplicação, a dosagem é reduzida, tendo rápida ação, baixo risco e efeito reversível (PEDRON, 2014a; SENESI, et al., 2015). O efeito da toxina pode ser observado ente uma e duas semanas, e normalmente dura entre quatro e seis meses (MOSTAFA, 2018).

Botox é uma técnica conservativa temporária efetiva, que melhora não só sorriso gengival, como o músculo que o envolve. Sendo considerada uma técnica segura para corrigir o display gengival, desde que usada em dose apropriada (ALFOUZAN et al., 2017). É produzida pelo Clostridium botulinum, bacilo anaeróbio, que pode causar infecção alimentar. Há sete sorotipos de toxina botulínica $(A, B, C 1, D$, E, F e G), com diferentes tempos de ação, toxicidade específica e potenciais de ação. Contudo, a mais utilizada é o sorotipo A (CÔRTE-REAL DE CARVALHO et al., 2011).

As principais vantagens são: a facilidade técnica, a alta tolerabilidade pelo paciente, o baixo índice de complicações e o efeito praticamente imediato e natural; sendo sua principal desvantagem a manutenção do resultado por um curto período de tempo (DA ESCÓSSIA et al., 2014). Em um procedimento bem realizado, os efeitos colaterais são mínimos, sendo a dosagem fundamental para o sucesso (POLO, 2008).

A injeção de toxina botulínica é considerada um tratamento invasivo mínimo de sorriso gengival, recomendado para pacientes cujos sorrisos gengivais são causados 
principalmente por músculos labiais hiperativos. Quando injetada por via intramuscular, a toxina cliva a proteína SNAP-25 associada ao sinaptossoma, bloqueando a liberação de acetilcolina e permitindo a repolarização do termo póssináptico, que produz desnervação química parcial do músculo, resultando na redução localizada das atividades do músculo elevador e relaxamento da ação de puxar para cima o lábio durante o sorriso (JASPERS et al., 2011).

Dentre as diversas aplicações, a toxina botulínica pode ser usada para fins estéticos com o objetivo de minimizar marcas de expressões e rugas, provocando a paralisia muscular da área. Na área de atuação do cirurgião-dentista, a toxina pode ser usada em casos de bruxismo, disfunções e dores na articulação temporomandibular, dor orofacial, queilite angular, sorriso assimétrico, hipertrofia de masseter, sialorréia, pós-operatório de cirurgias periodontais e de implantes, e nas dores de cabeça de origem dentárias (BUOSI et al., 2001; MARCIANO et al., 2014; OLIVEIRA et al., 2020).

Atualmente, o cirurgião-dentista é habilitado ao uso terapêutico e estético, de acordo com a Resolução CFO-176/2016 desde que não extrapole sua área anatômica de atuação (superiormente ao osso hióide até o limite do ponto násio e anteriormente ao trágus, abrangendo estruturas anexas) e para casos de procedimentos não cirúrgicos, inclui-se também o terço superior da face (OLIVEIRA et al., 2020).

Devido ao potencial minimamente invasivo, a rapidez e a eficácia que a toxina botulínica possui no tratamento de sorriso gengival, a determinação da origem do problema é extremamente importante, em pacientes que apresentam um sorriso gengival, que pode ser; esquelético, muscular, dentogengival ou uma combinação de vários ou mais fatores. Conhecer a origem do problema ajuda a orientar as decisões sobre o tratamento.

\section{REFERENCIAL TEÓRICO}

\subsection{SORRISO GENGIVAL}

É importante diferenciar o sorriso social e o sorriso espontâneo. O primeiro é um sorriso voluntário que o indivíduo faz quando se encontra em um ambiente social 
ou quando está a posar para uma fotografia. Neste, o lábio superior (LS) é elevado até ao nível do sulco nasogeniano, pela contração dos músculos elevadores do LS (elevador do lábio superior, elevador comum do lábio superior e da asa do nariz e zigomárico maior). O sorriso espontâneo, por sua vez, é involuntário e representa a emoção que o indivíduo está a experimentar no momento. Neste, há uma maior elevação de ambos os lábios e acentuação do sulco nasogeniano devido à ação, principalmente, dos músculos elevadores do LS, zigomático maior e fibras do músculo bucinador (SEIXAS et al., 2011; SARVER \& ACKERMAN, 2003).

Um importante parâmetro a avaliar quando da análise do sorriso é a linha do deste. Esta corresponde à quantidade de estrutura dentária vertical, que é visível durante o sorriso. Não é mais que a representação da relação entre o lábio superior e o incisivo central superior, quando ocorre o sorriso (SABRI, 2005).

O sorriso gengival é o termo utilizado para descrever uma condição estética relativamente frequente, caracterizada por uma exposição excessiva de gengiva ao nível da maxila, durante o sorriso (ALPISTE-ILLUECA, 2011; DINKER et al., 2014). ÉP portanto um termo descritivo, e não um diagnóstico. A população em geral considera uma exposição de, até $3 \mathrm{~mm}$, aceitável (ABOU-ARRAJ \& SOUCCAR, 2013).

A etiologia do sorriso gengival é multifatorial. Os fatores etiológicos podem ser de origem: gengival (erupção passiva alterada), muscular (hiperatividade do lábio superior ou lábio curto), esquelética (crescimento vertical maxilar excessivo), dentoalveolar (extrusão dento-alveolar anterior, sobre erupção compensatória) e/ou externa (induzida por placa bacteriana e por fármacos). É comum que o excesso de exposição gengival ocorra como resultado da interação de vários fatores. Estes fatores etiológicos podem surgir isoladamene ou em combinação (BIDRA et al., 2012; JANANNI et al., 2014).

Um adequado exame clínico e um correto diagnóstico são essenciais para determinar qual a causa do sorriso gengival. Desta forma, é possível demonstrar ao paciente quais são as abordagens terapêuticas mais adequadas, de modo a este opte de forma consciente. As várias opções de tratamento disponíveis incluem técnicas cujo fim é diminuir a quantidade de gengiva mediante a remodelação gengival e/ou óssea; técnicas que diminuem a altura óssea maxilar; reposicionamento dentário ortodôntico, ou técnicas cujo objetivo é reduzir a capacidade de elevação ou função 
do LS (ISHIDA, 2012).

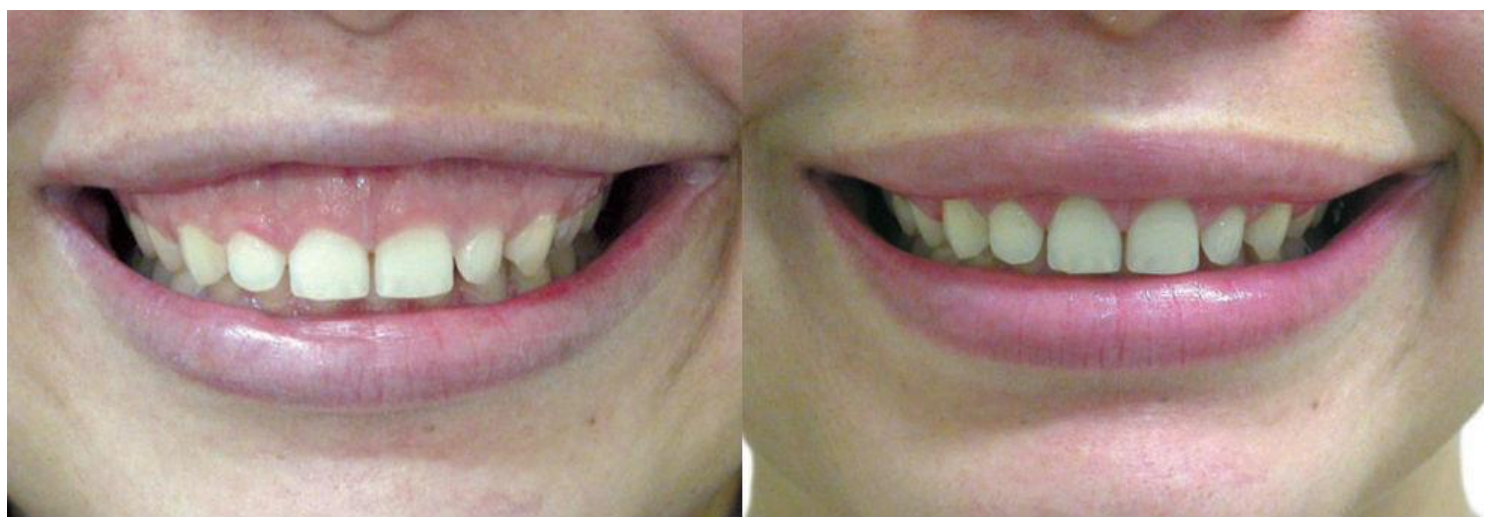

Figura 1 - Sorriso gengival antes e depois de tratamento. Fonte: Google

\subsection{TÉCNICAS DE CORREÇÃO}

As várias opções de tratamento disponíveis na literatura incluem técnicas cujo fim é reduzir a capacidade de elevação ou a função do lábio superior (reposicionamento labial, aplicação de toxina botulínica, respectivamente); diminuir a quantidade de gengiva mediante a remodelação gengival e/ou óssea (alongamento coronário); reposicionamento ortodôntico e reposicionamento superior do maxilar (MANTOVANI et al., 2016).

Como para qualquer técnica cirúrgica, alguns cuidados pré-operatórios devem ser executados, tal como: analgesia, assepsia e anestesia local e, antibioticoterapia profilática quando indicada. Após terminada a cirurgia é necessário minimizar: a dor, a inflamação e o risco de bactérias, de forma a tornar o pós-operatório o mais confortável possível. Indicações pós-operatórias específicas devem ser dadas ao paciente (MUTHUKUMAR et al. 2015).

A técnica de reposicionamento labial tem como objetivo, minimizar a quantidade de exposição gengival durante o sorriso. Para tal, o lábio superior é reposicionado numa localização mais apical à original o que acaba por limitar a retração dos músculos elevadores do sorriso (DAYAKAR et al., 2014).

O alongamento coronário consiste em um procedimento cujo objetivo é expor a estrutura dentária saudável, tendo como propósito a possibilidade de restaurar o dente reestabelecendo, desta forma, a sua função ou, um propósito estético, no qual o excesso de exposição gengival interfere na harmonia do sorriso do paciente. Várias 
técnicas são para a realização do alongamento coronário: gengivectomia, mediante a realização de um retalho reposicionado apicalmente com ou sem resseção de tecidos moles e duros, ou através da extrusão ortodôntica forçada (MAJZOUB et al., 2014).

A gengivectomia é realizada quando existe quantidade de tecido gengival suficiente para que, após a sua remoção, a margem gengival livre fique a $3 \mathrm{~mm}$ da crista óssea alveolar. Para a realização de gengivectomia deverão estar presentes três principais condições: (1) localização da crista alveolar óssea em relação à JAC de, aproximadamente, 1,5 a $2 \mathrm{~mm}$, (2) espessura adequada de osso alveolar e (3) banda de gengiva queratinizada larga. Caso não se verifiquem estas condições, é provável que ocorram recidivas (ABOU-ARRAJ \& SOUCCAR, 2013).

O retalho posicionado apicalmente (RPA) consiste na elevação de um retalho muco-periósteo de espessura total que é posicionado numa localização mais apical em relação à posição inicial. Este tipo de procedimento está mais indicado quando a largura de gengiva queratinizada é mínima (menor ou igual que $3 \mathrm{~mm}$ ), de forma a preservá-la ao máximo possível (ROSSI et al., 2014).

O retalho posicionado apicalmente com resseção óssea é uma técnica de alongamento coronário mais realizada uma vez que, na maioria dos casos, a resseção óssea é requerida. Para a realização de resseção óssea uma das seguintes condições deverá estar presente: estreita proximidade entre a crista óssea alveolar e a JAC e/ou fina espessura de osso alveolar. Este procedimento combina ambos os passos da gengivectomia e do RPA. Antes de iniciar a resseção óssea, a posição do osso alveolar em relação à margem gengival é determinada (ABOU-ARRAJ \& SOUCCAR, 2013).

A toxina botulínica quando aplicada nos músculos responsáveis pela elevação do lábio superior, ocorre uma inibição da contração dos mesmos o que permite a redução da exposição gengival quando sorrindo. Simultaneamente, pode ocorrer uma diminuição do sulco nasolabial (DINKER et al., 2014).

\subsection{HISTÓRICO DA UTILIZAÇÃO MÉDICA DA TOXINA BOTULÍNICA}

A toxina botulínica é um complexo proteico purificado, de origem biológica, obtido a partir da bactéria Clostridium Botulinum, gram-positiva, anaeróbica, formadora de esporos, presente especialmente no solo e que em condições favoráveis 
para sua reprodução, produz sete sorotipos diferentes (A, B, C, D , E, F e G), dentre eles, os sorotipo mais utilizado é o do tipo A (BENECKE, 2012).

$\mathrm{Na}$ década de 1900 devido ao surto de botulismo nos EUA, pesquisas se intensificaram e em 1920, na Califórnia, isolaram a neurotoxina. Mas apenas em 1946 a toxina na forma cristalina foi desenvolvida e purificada por Dr. Edward J. Schantz e Dr. Erik A. Johnson em associação com o Dr. Carl Lamanna (SPOSITO, 2009). Diante dessas descobertas, pesquisadores sugeriram o uso da toxina Botulínica (toxina botulínica) para o tratamento da hiperfunção muscular e, no final dos anos sessenta, iniciaram uma pesquisa observada em alguns primatas utilizando a toxina botulínica tipo A nos músculos oculares para o tratamento do estrabismo (CAZUMBÁ et al., 2017; OLIVEIRA et al., 2020).

Deste então, o uso cosmético da toxina botulínica tipo A evoluiu e se expandiu em todo mundo. No ano de 1988 ela foi utilizada pela primeira vez em tratamento estético para diminuir rugas faciais e linhas de expressão, com o resultado foi muito satisfatório. No entanto, só em 1989 a toxina botulínica A (sorotipo mais utilizado) foi aprovada pela Food and Drug Administration (FDA) para fins terapêuticos que envolvessem distúrbios do movimento e, no ano seguinte, o consenso do National Institutes of Health (NIH) aprovou a toxina botulínica como um medicamento seguro e eficiente (SPOSITO, 2009; OLIVEIRA et al., 2020).

Carruthers \& Carruthers (1992) descobriram que a toxina reduz o aparecimento de rugas na região glabelar, resultado do relaxamento dos músculos que controlam as expressões faciais, também sendo eficaz nas rugas ao redor dos olhos e nas dobras nasolabiais.

Desde então, o escopo da aplicação da toxina botulínica expandiu-se para o tratamento de distúrbios oftalmológicos, como blefarospasmo e distúrbios neurológicos, como espasmos faciais e distonia cervical e de membros, e tem sido usado principalmente para aliviar a tensão inadequada ou excessiva nos músculos esqueléticos (PARK, et al., 2016).

A utilização dessa toxina purificada em procedimentos cosméticos só foi aprovada pela Anvisa, no Brasil, em 2000 e, nos EUA, pela FDA, em 2002, e desde então seu uso sucessivo vem auxiliando no tratamento de diversas patologias (PEDRON, 2015). Na Odontologia brasileira a toxina botulínica-A foi devidamente regulamentada para uso pela resolução 112/11 do Conselho Federal de Odontologia 
desde setembro de 2011 (BRASIL, 2011).

O Brasil é considerado o segundo no ranking de comercialização da toxina botulínica, perdendo apenas para os EUA. Movimentando mais de 100 milhões de reais por ano, $80 \%$ da toxina importada, é destinada para o uso cosmético e os $20 \%$ restantes, são utilizados para uso terapêutico. O gasto com a importação da toxina chega a US\$ 7 milhões, com gastos aplicados em mais de U\$3 milhões em injeções de toxina botulínica ao ano (PERÃO \& BARBOSA, 2015).

Atualmente, o uso da toxina na área da odontologia está cada vez mais crescente. Isso se deve à grande procura por parte dos pacientes que visam melhorar a estética e aparência física. Antigamente, a toxina botulínica era utilizada somente para tratamento de estrabismo e outras doenças, porém com os avanços na tecnologia e ciência, outras formas de utilização foram surgindo, principalmente na área da estética orofacial. De aplicação fácil, é um método simples, não cirúrgico e indolor. Uma especialidade nova que a cada ano inova suas técnicas e seus avanços.

\subsubsection{MARCAS COMERCIAIS DISPONÍVEIS}

Botox é o nome popular dado à marca da toxina botulínica tipo $\mathrm{A}$, produzida pela empresa farmacêutica americana Allergan. No brasil a marca Botox foi aprovada em 1992 para indicações terapêuticas e, em 2000 para o tratamento de rugas e hiperidrose axilar e palmar. Por ser a primeira aprovada, o botox tornou-se muito conhecida, e por isso virou sinônimo do procedimento.

Há no mercado também, outras marcas como a BOTULIFT, (AMGEM / Bergamo), produzida pela farmacêutica Sul coreana Medtox empresa conceituada e de referência na fabricação desse medicamento. É uma das marcas mais vendidas e utilizadas no meio estético. E temos outras marcas menos conhecidas como: XEOMIN, disponibilizado em frascos de 100U, apenas na forma liofilizada; PROSIGNE, disponibilizado $50 \mathrm{U}$ e 100U, sempre na forma liofilizada; DYSPORT, disponibilizado em frascos de $300 \mathrm{U}$ e 500U, apenas na forma liofilizada; BOTULIM, disponibilizado em frascos de 50U, $100 \mathrm{U}$ e $200 \mathrm{U}$.

\subsection{FARMACOLOGIA DA TOXINA BOTULÍNICA}

O mecanismo de ação da toxina botulínica se dá na clivagem da proteína 
associada ao sinaptossoma (SNAP-25) e inibição da liberação de acetilcolina (ACh), impedindo a contração muscular. Existem sete tipos sorologicamente distintos de toxina botulínica disponíveis, dos quais o tipo A (toxina botulínica-A) parece ser o mais potente e é mais frequentemente utilizado na prática clínica (DINKER et al., 2014). Em estudo de Polo (2008), foi demonstrado que o uso da toxina botulínica-A é uma técnica eficaz e minimamente invasiva para obter a redução na exibição gengival em pacientes, com hiperfunção do músculo elevado do lábio.

O botox é formado por uma cadeia de polipeptídios de 150.000 daltons, sendo subdividido em duas cadeias; uma leve de 50.000 daltons e uma pesada de 100.000 daltons, ligadas por pontes, impedindo que se torne tóxica e, portanto, permitindo a sua utilização terapêutica (FAVRE-GUILMARD, et al., 2017).

\subsubsection{TRANSMISSÃO NEUROMUSCULAR}

A transmissão neuromuscular colinérgica envolve várias etapas como: a síntese, armazenamento, libertação, ligação, degradação e reciclagem de acetilcolina. Durante a síntese, a colina é transportada a partir do fluido extracelular para o citoplasma do neurónio colinérgico por um sistema de cotransporte com sódio e já dentro da célula reage enzimaticamente com acetil-CoA para formar a acetilcolina, sendo depois armazenada em vesículas sinápticas em forma de grânulos. No terminal nervoso da junção neuromuscular há várias vesículas contendo os neurotransmissores. Com a chegada do potencial de ação, as vesículas viajam até ao terminal da junção nervosa, os canais de cálcio voltagem dependentes da membrana pré-sináptica abrem, provocando um aumento na concentração de cálcio intracelular (WHEELER \& SMITH, 2013; HUANG, 2000).

Os níveis de cálcio elevados promovem a ancoragem e fusão das vesículas sinápticas com a membrana celular através de um mecanismo complexo que envolve várias isoformas da proteína SNARE, culminando na liberação de acetilcolina, que vai ligar a receptores da membrana pós-sináptica permitindo a contração muscular (HUANG, 2000).

A toxina botulínica (BTX) causa denervação química temporária de músculos esqueléticos, por bloqueio da liberação mediada por $\mathrm{Ca}^{2}{ }^{2}$ de acetilcolina, das terminações nervosas de neurônios motores alfa e gama, produzindo um 
enfraquecimento dose-dependente, temporário da atividade muscular tornando os músculos não funcionais sem que haja efeitos sistêmicos. Entretanto acredita-se que o músculo inicia a formação de novos receptores de acetilcolina. À medida que o axônio terminal começa a formar novos contatos sinápticos, há um reestabelecimento da transmissão neuromuscular e retorno gradual à função muscular completa, geralmente com efeitos colaterais mínimos (CARRUTHERS \& CARRUTHERS, 2004).

\subsubsection{MECANISMO DO BLOQUEIO NEUROMUSCULAR}

A toxina botulínica tipo A bloqueia a transmissão da sinapse colinérgica, reduzindo assim, a atividade muscular. Há muito se sabe que a toxina botulínica-A pode ser usada para melhorar a aparência estética das linhas faciais, com efeitos clínicos tornando-se evidentes em poucos dias e atingindo o efeito máximo em 2 semanas. Os resultados geralmente duram 4 meses a 6 meses (INDRA et al., 2011).

Em relação ao seu mecanismo de ação, a cadeia pesada se une aos terminais nervosos da junção neuromuscular e a cadeia leve é transportada, para o interior do terminal nervoso, e assim bloqueia a liberação da acetilcolina na membrana présináptica causando fraqueza muscular (MAZZUCO \& HEXSEL, 2010; FAVREGUILMARD, et al., 2017), pois para que haja contração muscular é necessário que a acetilcolina seja liberada na placa motora, devido a esse mecanismo de ação, quando ela é infiltrada nos músculos causa uma redução da contração do mesmo, ocorrendo então uma menor exposição gengival (MAZZUCO \& HEXSEL, 2010).

A figura, a seguir mostra detalhadamente o mecanismo de ação da toxina quando injetada no músculo: 


\section{O MECANISMO DE AÇÃO DA TOXINA BOTULÍNICA}

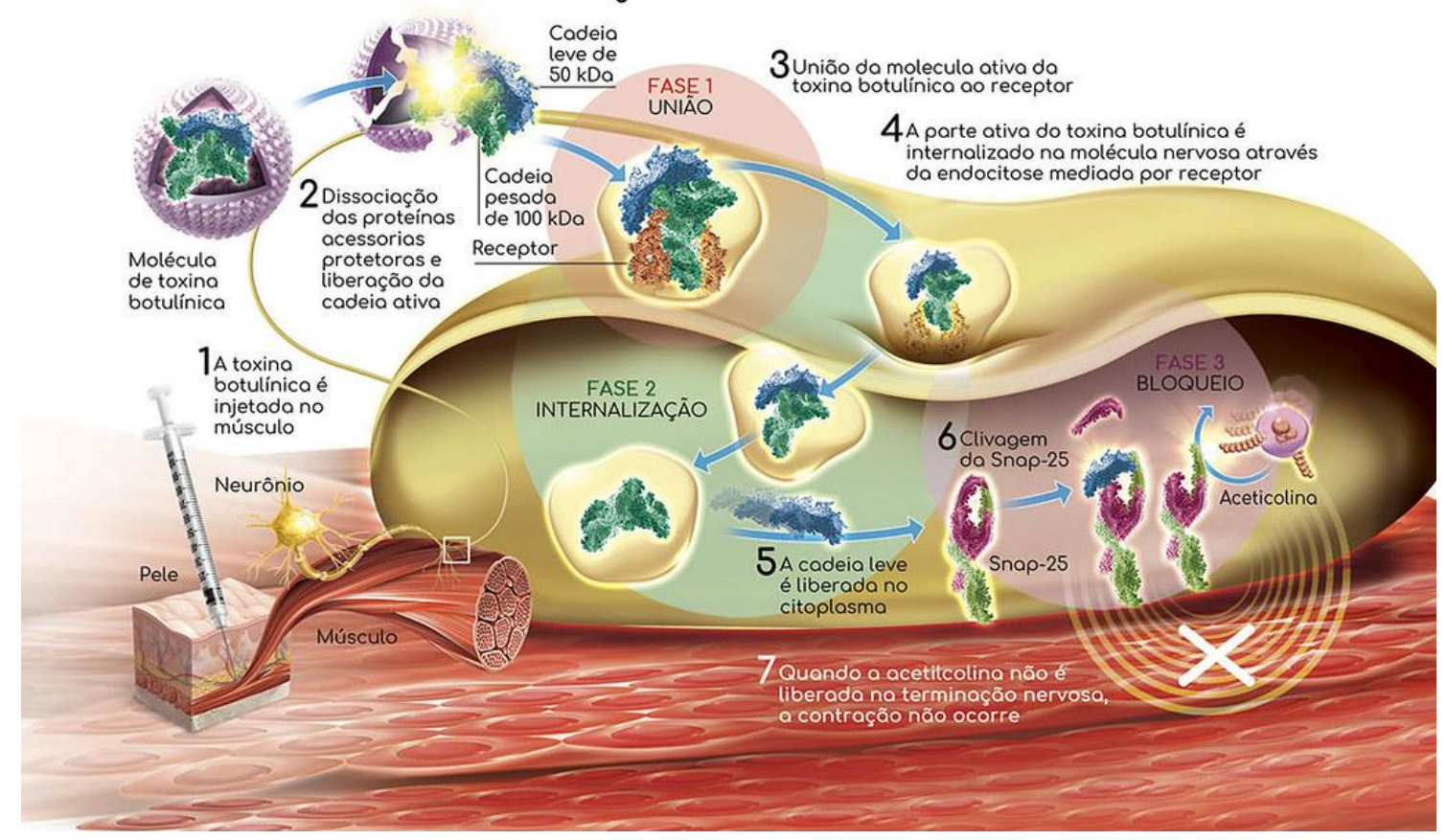

Figura 2- Mecanismo de Ação da Toxina Botulínica. Fonte: Google

O complexo SNARE é o local de ação para a toxina botulínica. Caso uma ou mais proteínas SNARE sejam danificadas, as vesículas de acetilcolina não vão conseguir ligar-se e fundir-se à membrana pré-sináptica (Tighe \& SCHIAV, 2012; WHEELER \& SMITH, 2013). A estrutura primária da toxina botulínica é tal que as formas resultantes, das estruturas secundárias e terciárias criam uma ligação altamente específica, bloqueando a libertação da acetilcolina nas junções mioneurais ao nível do sistema nervoso periférico (SCHANTZ \& JOHNSON, 1992).

A toxina botulínica exerce o seu efeito neurotóxico, por um mecanismo de várias etapas como: a ligação, internalização, o tráfego intracelular, translocação da membrana e degradação proteolítica de alvos intracelulares específicos (TIGHE \& SCHIAVO, 2012). Intracelularmente, estas toxinas interferem com o processo de exocitose de vesículas sinápticas regulada por $\mathrm{Ca2}+$ e posterior libertação dos seus conteúdos para a fenda sináptica. O fundamental para que esta libertação do neurotransmissor se dê, é a fusão da vesícula sináptica com a membrana plasmática pré-sináptica, e é aqui que a toxina botulínica atua, inibindo este processo (WHEELER \& SMITH, 2013). 


\subsection{INDICAÇÕES E CONTRAINDICAÇÕES DA UTILIZAÇÃO DA TOXINA BOTULIINICA NA ÁREA ODONTOLÓGICA}

Neurotoxinas como a toxina botulínica (BTX) são documentadas no consultório dermatológico, para tratamento de: quelóides, cicatrizes hipertróficas, pênfigos como dermatose, genodermatose, acne inversa, psoríase, eritema facial, entre outros. Assim, a toxina botulínica tipo A (BTX-A) também tem sido utilizada para o manejo eficaz da musculatura hiperfuncional (NIGAM \& NIGAM, 2010).

A toxina botulínica é contraindicada em: gestantes e lactantes, casos de doenças neuromusculares, pacientes alérgicos aos componentes do produto, pacientes fazendo uso de aminoglicosídeos, anti-inflamatórios, anticoagulantes e drogas que atuam na junção neuromuscular, com hipersensibilidade a lactose e albumbina, e portadores de doença autoimune (KLEIN, 2001; CÔRTE-REAL DE CARVALHO et al., 2011; ROCHA, 2011).

As contraindicações para o bloqueio com toxina botulínica tipo A são classificadas em absolutas e relativas. As absolutas são alergia conhecida ao medicamento ou a seus componentes, infecção no sítio do bloqueio, gravidez e aleitamento, expectativa irreal do paciente e instabilidade emocional. Já as contraindicações relativas são: doença neuromuscular associada (síndrome póspólio, miastenia gravis, esclerose lateral amiotrófica); pessoas que necessitam da expressão facial; coagulopatia associada e/ou descompensada; doença autoimune em atividade; falta de colaboração do paciente para o procedimento global e uso de potencializadores como aminoglicosídeos em até quatro semanas antes do procedimento (SPOSITO, 2004).

As complicações possíveis do tratamento com toxina botulínica são divididas entre: relativas, raras e descritas. As complicações com risco relativo são: dor, hematoma, sensação de perda de força, sintomas gripais e gastrintestinais e infecção local. As complicações raras são: alergia - erupção de pele difusa (anafilaxia não descrita); atrofia focal; diplopia, dificuldade de acomodação visual; formação de anticorpos (3-5\%); sudoração alterada. Já as complicações descritas são: ptose de pálpebra e de sobrancelhas; disfagia; alteração da expressão ou face paralisada (máscara); assimetria; alteração funcional; fraqueza muscular intensa ou 
generalizada. As complicações relativas são evitáveis ou facilmente resolvíveis; as raras realmente têm incidência muito baixa, porém a formação de anticorpos é um efeito altamente indesejável e requer cuidados especiais por parte do médico. As complicações descritas, por sua vez, normalmente se devem a erro de técnica, erro na avaliação clínica e funcional do paciente para o procedimento, erro de dose ou de diluição (SPOSITO, 2004).

Silva (2009) contraindica a toxina botulínica $A$, em pacientes com doenças do sistema nervoso periférico ou com desordens neuromusculares, em coadministração de antibióticos que contêm aminoglicosídeos ou outros agentes, que interferem na transmissão neuromuscular, uso no tratamento de pacientes com processos inflamatórios presentes na pele e no local em que é realizada a aplicação. As complicações podem ser classificadas em dois grupos: as que provêm da injeção e as decorrentes do próprio produto. As derivadas da injeção são: edema e eritema, dificuldade de acomodação visual, ptose e dor ao elevar a pálpebra e agravamento das rugas. As reações adversas mais frequentes são as dores de cabeça, a disfagia e a hipersensibilidade.

\subsection{ANATOMIA DA MUSCULATURA DA BOCA}

A atividade de dar um sorriso é realizada por vários músculos faciais, como: 0 elevador do lábio superior, da asa do nariz, zigomático maior e menor, do ângulo da boca, orbicular da boca e risório. Entre os músculos citados, o elevador do lábio superior, da asa do nariz e zigomático maior, possuem a principal função de determinar a quantidade de elevação labial no sorriso gengival. Portanto, são os músculos mais afetados pela injeção da toxina. Algumas fibras dos destes se convergem para a mesma área, formando um triângulo, momento em que formará um ponto de eleição adequado com os três músculos (elevador do lábio superior, asa do nariz e zigomático maior) para se realizar a aplicação da toxina (PEDRON, 2015).

A figura abaixo mostra a região em fica cada músculo da face, para a aplicação da toxina, na correção do sorriso gengival: 


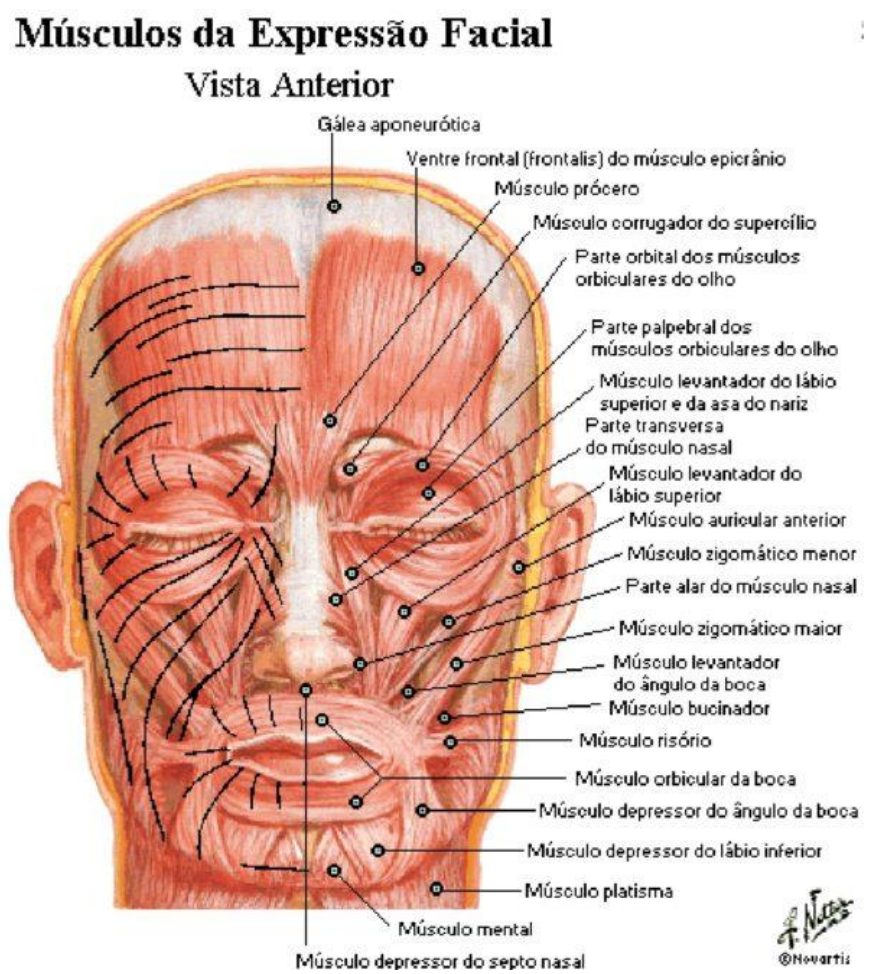

Figura 3 - Anatomia da musculatura facial. Fonte: Google.

Cada um dos músculos envolvidos na elevação do lábio superior apresenta algumas funções durante a atividade de realizar um sorriso gengival. Os locais para as injeções são determinados pela contração que o musculo realiza, estas resultam em diferentes áreas de visualização gengival. Diversas classificações foram propostas ao sorriso gengival como, por exemplo: anterior, posterior, misto e assimétrico, envolvendo grupos musculares diferentes (MAZZUCO \& HEXSEL, 2010).

O sorriso gengival anterior deve ser tratado com a técnica convencional, com aplicações lateralmente na asa do nariz. Nos pacientes com sorriso gengival posterior, a aplicação da toxina deve envolver os músculos zigomáticos maior e menor, com aplicação da toxina, em dois pontos diferentes: no ponto de maior contração do sulco nasolabial durante a atividade do sorriso, e o segundo ponto, $2 \mathrm{~cm}$ lateralmente ao primeiro, ao nível da linha do tragus. Aos pacientes que apresentam sorriso gengival misto, a aplicação da toxina deve ser realizada em todos os pontos mencionados acima (MAZZUCO \& HEXSEL, 2010).

\subsection{DOSES DE TOXINA BOTULÍNICA UTILIZADAS PARA O TRATAMENTO DE SORRISO GENGIVAL}


A toxina botulínica é uma neurotoxina produzida pelo Clostridium botulinum, e possui uma dose letal média (DL50 - dose de toxina capaz de levar à morte $50 \%$ da população a ela exposta) de 1 nanograma de toxina por quilograma de peso corporal (10-9 g/kg), dessa maneira vai depender da dosagem para identificá-la como um veneno ou não (BACHUR, 2009).

Os protocolos atuais de aplicação da BTX-A preconizam a aplicação de $10 \mathrm{U}$ bilateralmente na região do sulco nasolabial adjacente à asa do nariz. Doses adicionais de BTX-A poderão ser realizadas entre 15 e 30 dias da primeira aplicação no intuito de corrigir eventuais assimetrias. Nesses casos, a dose recomendada é de 5 U no lado de maior contração muscular (KUHN-DALL'MAGRO, 2015)

Kuhn-Dall'Magro et al. (2015) citam o seguinte protocolo de tratamento: primeiro se deve fazer a higienização da face, seguido de demarcação (com lápis tinta). Deve-se então realizar a anestesia com anestésico tópico - EMLA (lidocaína 25 $\mathrm{mg} / \mathrm{g}$; prilocaina $25 \mathrm{mg} / \mathrm{g}$ AstraZeneca), para então seguir com a aplicação do produto na área demarcada; 10 U de cada lado. Após a aplicação é recomendado manter a cabeça elevada por 4 horas e não comprimir a região e nem realizar atividades físicas por 24 horas. O retorno deve ocorrer entre 15 e 30 dias após a aplicação.

Para Moreira et al. (2019), antes da injeção da toxina botulínica, a superfície da pele deve ser limpa com álcool etílico a 70\% para remoção da oleosidade. Para o Botox $100 \mathrm{U}$ em pó, diluir em $2 \mathrm{ml}$ de solução salina 0,9\% estéril, sem conservante. Usando uma seringa de tuberculina calibrada de 1,0 ml com manômetro de 26 a 30 manômetros, injetar no local recomendado 2.5U. Após a aplicação, o paciente não deve manipular a área tratada, não deitar a cabeça ou realizar atividades físicas nas primeiras 4 horas após submetido ao procedimento. Deve-se observar durante os próximos 7 e 15 dias a resposta ao procedimento.

\subsection{EFEITOS COLATERAIS E COMPLICAÇÕES DA TOXINA BOTULÍNICA}

O uso do BTX-A para gerenciar a alteração do sorriso depende da compreensão do dentista, em todos os aspectos da anatomia regional da face. Assim, os efeitos do BTX-A são sensíveis à técnica e podem causar várias complicações e resultados adversos (DELPACHITRA et al 2018). Após a injeção da toxina, esta difunde-se para os músculos e outros tecidos. O seu efeito diminui com o aumento da 
distância do local da injeção contudo, quando são injetados grandes volumes, podese espalhar para músculos adjacentes (MÜNCHAU \& BHATIA, 2000).

O grau de toxicidade da TBA é resultante da atividade catalítica da cadeia $L$, (que é uma zinco-endopeptidase) e da ligação dissulfídrica. A cadeia $\mathrm{H}$ liga-se às proteínas existentes na membrana sináptica, e faz com que a cadeia $L$ entre na célula e clive uma proteína específica, em um local específico. Porém, se a ligação dissulfídrica for quebrada, antes da internalização da toxina na célula, a cadeia $L$ não vai ser capaz de conseguir penetrar na membrana sináptica do terminal do axônio, havendo uma perda total de toxicidade, ou seja, perda do efeito da toxina botulínica (SILVA, 2009; COLHADO, et al., 2009).

De acordo com Delpachitra et al (2018) os resultados adversos mais comuns do uso de BTX-A no sorriso gengival são: assimetria, sobrecorreção, subcorreção e queda perioral. Além disso, os pacientes podem ficar menos satisfeitos com os resultados dinâmicos, do que com os observados em repouso.

Devido ao uso da toxina ser via intramuscular, a aplicação pode causar dor no local e sensibilidade anormal e em alguns casos infecionar, também é possível ocorrer: parestesia facial, fraqueza muscular, alterações salivares e dificuldades na fala. Apesar de ser um procedimento simples e seguro, a injeção da toxina botulínica associar-se a alguns eventos adversos como: dor no local da injeção, hematomas, infecção, edema, disfonía, disfagia, ptose, alongamento do lábio superior e assimetria do sorriso. O cirurgião-dentista deve estar atento, em relação a posologia, precisão da técnica e localização da puntura (POLO, 2005; NIAMTU, 2008; MAZZUCO \& HEXSEL, 2010; JASPERS, et al., 2011; PEDRON, 2014a).

Entre as sérias complicações que podem ocorrer estão: comprometimento das vias aéreas, dificuldade de mastigação e deglutição. O uso repetido da toxina botulínica-A para fins cosméticos não está associado a um aumento nos resultados adversos ou alterações no perfil de segurança (ALMASRI, 2015).

Em estudo randomizado de Zagui et al. (2008) com 1003 pacientes, encontrouse a seguinte porcentagem de efeitos adversos: ptose palpebral (3\%), olho seco (2.29\%), edema local (1,99\%), boca seca (1,99\%), cefaléia (1,59\%).

As contraindicações da utilização da toxina botulínica são gestação; lactação; hipersensibilidade à própria toxina botulínica, lactose e albumina; doenças musculares e neurodegenerativas (miastenia gravis e doença de Charcot); e uso simultâneo de 
antibiótico aminoglicosídico, que pode potencializar a ação da toxina (JASPERS et al., 2011; PEDRON, 2014b).

Com a utilização da toxina, alguns efeitos indesejáveis podem ocorrer dependendo da quantidade injetada e o local de aplicação. São efeitos temporários e reversíveis, que possuem duração de semanas, podendo em alguns casos chegar a meses. É improvável que os resultados sejam perfeitos, portanto as expectativas dos pacientes também devem ser gerenciadas, sendo que, a maioria das complicações é leve e de curta duração (DELPACHITRA et al 2018).

\subsection{ASPECTOS IMUNOLÓGICOS}

Semelhante a todos os antígenos estranhos, a toxina botulínica também provoca a produção de anticorpos, que inibem seu efeito terapêutico ao induzir reações imunológicas no organismo. Desde que são proteínas estranhas ao organismo humano, anticorpos podem ser formados contra a porção tóxica ou contra as suas proteínas, não tóxicas. A exposição a antígenos de toxina botulínica estimula uma resposta imune ativando: linfócitos $\mathrm{B}$ e $\mathrm{T}$, células de memória imune, formação de citocinas e finalmente, a formação de anticorpos (WENZEL, 2004).

Os anticorpos bloqueiam a atividade biológica e induzem a falha terapêutica. Estes são chamados de neutralizantes ou bloqueadores, sendo formados contra as porções proteicas não tóxicas são chamados não neutralizantes (DRESSLER \& BENECKE, 2007). Os anticorpos neutralizantes se ligam aos antígenos da toxina botulínica diminuindo sua efetividade e as células de memória irão ser acionadas provocando respostas imunes em aplicações sequenciais. Este fato tem alta relevância clínica, uma vez que repetidas aplicações são normalmente necessárias, para o tratamento de afecções crônicas (WENZEL, 2004).

Embora tenha sido relatado que $40-60 \%$ dos pacientes produzem anticorpos, durante o tratamento com BTX-A, aqueles que inibem os efeitos terapêuticos são produzidos apenas em 2-5\% dos pacientes (KESSLER, et al., 1999; CRITCHFIELD, 2002). Os fatores que aumentam os riscos de produzir anticorpos neutralizantes durante o tratamento com BTX-A incluem injeção frequente de BTX-A durante um curto período, injeções em altas doses e aumento da dose de injeções (NAUMANN, et al., 2006). Além disso, em estudos de Dressler \& Eleopra (2006), foram relatados 
uma maior produção de anticorpos com BTX-B do que com BTX-A.

Os fatores de risco para a falha terapêutica associada a anticorpos, incluem a dose de tratamento e o intervalo entre as aplicações sucessivas. A importância da dose reside na correlação, com a carga proteica injetada, e este fato está relacionado com a formulação utilizada. O risco não está associado a atividade biológica em si, mas com a quantidade e a frequência com que o antígeno se apresenta ao sistema imune (DRESSLER \& BENECKE, 2007).

A toxina botulínica possui propriedade altamente imunogênica, com isso ocorre o desenvolvimento e formação de anticorpos. Se o profissional aplicar doses elevadas na administração ou ocorrer aplicações com frequência sem respeitar os intervalos de tempo, ocorrerá a diminuição da função da toxina com o passar do tempo. Em diversas ocasiões a mudança para outro subtipo de toxina, pode ser imprescindível. A toxina tipo B é menos utilizada nos procedimentos quando comparada ao tipo A, devido este tipo ser mais imunogênica, ou seja, na obtenção de um resultado satisfatório exige-se doses cada vez maiores, acarretando uma maior carga de proteínas e por efeito elevando sempre a mais seu potencial imunogênico (DUTRA MB, et al., 2011)

\section{APLICAÇÃO DE TOXINA BOTULÍNICA E SORRISO GENGIVAL}

O uso da toxina botulínica é muito diversificado, possibilitando ser usada em pacientes com alterações faciais e alterações relacionadas à saúde bucal, proporcionando bons resultados finais, quando em comparação com opções de tratamento adicionais. Na odontologia, o uso da toxina se tornou relevante, por ser um método não invasivo e uma opção de tratamento seguro, substituindo procedimentos cirúrgicos (GRACCO \& TRACEY, 2010).

A preparação da toxina é específica para cada marca e o profissional deve ler atentamente as instruções para o uso, para assim saber exatamente a proporção que deve ser usada. O produto é vendido em frascos contendo $100 \mathrm{U}$ ou $50 \mathrm{U}$ de toxina em pó nas formas liofilizada, estéril e a vácuo. O uso deve ser feito no menor período de tempo e a toxina deve ser armazenada em geladeira, entre $2^{\circ} \mathrm{C}$ e $8^{\circ} \mathrm{C}$, por até a 4 horas (SRIVASTAVA et al., 2015). Armazenar o produto já diluído pode ocasionar falta de eficácia ao longo do tempo (BENEDETTO, 1999). A comercialização do produto em forma a vácuo é segura e recomenda-se não usar formas não a vácuo. 
No entanto, devido ao vácuo, deve-se tomar cuidado durante a diluição, impedindo que a ingestão de solução salina ocorra rapidamente, causando a agitação da droga, o que pode quebrar as moléculas (SPOSITO, 2004).

A toxina botulínica atua causando relaxamento muscular, por meio do bloqueio da acetilcolina, um neurotransmissor, que transporta mensagens do cérebro para as fibras musculares, impedindo assim, a contração muscular (MOREIRA et al., 2019). A toxina botulínia tipo A tem sido amplamente utilizada na odontologia, para o tratamento de hiperfunção muscular, como é o caso do sorriso gengival, além de desordens temporomandibulares, assimetrias faciais, hipertrofia massetérica, espasmo hemifacial, dor miofascial, sialorréia e bruxismo (SANDLER, et al., 2007; EMARA, et al., 2013).

A grande vantagem desse tipo de tratamento é que é $100 \%$ reversível, havendo assim, reversão na aparência caso o paciente não esteja contente, devido ao efeito temporário (SANDLER, et al., 2007). Além disso, a latência média do tempo da injeção para o início da manifestação clínica é de três a cinco dias e a duração do benefício é, geralmente, de três a quatro meses (JANKOVIC, 2004).

O contorno labial é estabelecido pela ação dos músculos faciais, tais como: levantador do lábio superior e asa do nariz (LLSAN), levantador do lábio superior (LLS), e os músculos zigomáticos menor (ZMi) e maior (ZMj)3,4. Dentre esses, o LLSAN, o LLS, e o ZMi estabelecem a quantidade de elevação labial, que ocorre quando o paciente sorrir, sendo, portanto, os músculos-alvo para a ação da toxina botulínica (FAVRE-GUILMARD, et al., 2017).

\section{CONSIDERAÇÕES FINAIS}

A toxina botulínica pode ser uma alternativa segura e menos invasiva para tratamento de sorriso gengival provocado pela hiperatividade dos músculos elevadores do lábio superior. A neurotoxina apresenta uma vasta aplicação nas ciências da saúde, com destaque para a odontologia e em diversos tipos de tratamentos funcionais e estéticos.

Apesar da toxina ser uma prática recente na odontologia, se mostra uma ferramenta poderosa e de grande importância, nos tratamentos de sorrisos gengivais, que levam a altos níveis de satisfação no paciente, podendo assim, ajudar a melhorar 
a qualidade de vida e ao mesmo tempo, a autoestima dos pacientes. Sua utilização requer conhecimento para compreender os efeitos que a toxina pode causar em nível local e sistêmico, assim como: riscos, benefícios e responsabilidade ética.

Por possuir conhecimento sobre as estruturas de cabeça e pescoço o cirurgiãodentista pode tratar certas afecções da face e da cavidade oral de forma conservadora e segura com a aplicação da toxina botulínica, desde que possua treinamento específico e conhecimento, sobre a sua utilização e não extrapole suas funções.

Neste sentido há necessidade de a toxina ser administrada por profissionais qualificados e que tenham respaldo legal, para o seu uso. A toxina botulínica é portanto, um complemento útil na melhora estética do sorriso gengival, podendo ser mais uma opção terapêutica ao alcance do periodontista, na finalização e complementação dos seus casos. 


\section{REFERÊNCIAS}

ABOU-ARRAJ, R.V.; SOUCCAR, N.M. Periodontal treatment of excessive gingival display. Seminars in Orthodontics, 2013. v. 4, p. 267-278

AL-FOUZAN, A. F.; MOKEEM, L. S.; AL-SAQAT, R. T.; ALFALAH, M. A.; ALHARBI, M. A.; AL-SAMARY, A. E. Botulinum Toxin for the Treatment of Gummy Smile. The Journal of Contemporary Dental Practice, 2017. v. 18, n. 6, p. 474-478.

ALMASRI, M. Surgical Techniques to Improve the Smile. A Textbook of Advanced Oral and Maxillofacial Surgery, 2015. v.2, p. 191-206.

ALPISTE-ILLUECA, F. Altered passive eruption (APE): A little -known clinical situation. Med Oral Patol Oral Cir Bucal, 2011. v.16, p. 100-4.

BACHUR, T.P.R.; SOUSA, F.C.F.; SOUZA, M.M.C.; VASCONCELOS, S.M.M.; VERÍSSIMO, D.M. Artigo de Revisão Toxina Botulínica: de Veneno A Tratamento. Revista Eletrônica Pesquisa Médica, 2009; v.3, n.1.

BENECKE, R. Clinical relevance of botulism toxin immunogenicity. BioDrugs, 2012. v.26, n.2, p. 1-9.

BENEDETTO, A.V. The cosmetic uses of Botulinum toxin type A. Int $\mathbf{J}$ Dermatol. 1999, v.38, n. 9, p. 641-55.

BIDRA, A.V.; AGAR, J.R.; PAREL, S.M. Management of patients with excessive gingival display for maxillary complete arch fixed implant-supported prostheses. $\mathbf{J}$ Prosthet Dent, 2012. v. 8, p. 324-31.

BRASIL. Resolução CFO-112/2011. Baixa normas sobre a utilização da toxina botulínica e ácido hialurônico. Setembro 2011 [acesso em: 15 abr 2020].

BUOSI, M.B.; CARVALHO L.G.; CUBO, R.; FABRÍCIO, B.; IANELI L. C.; OLIVO J.Z.O. Uso da Toxina Botulínica na Odontologia. Anais do Fórum de Iniciação Científica da Funec. 2011.

CARRUTHERS, J.D.; CARRUTHERS, J.A. Treatment of glabellar frown lines with c. Botulinum-a exotoxin. J Dermatol Surg Oncol. 1992, v. 18, p.17-17

CAZUMBÁ, F. B, .;SÁ, R. C, ; KALIL, M.T.A. C.; KALIL, M. V.; FERNANDES, G. V. O. Uso de Toxina Botulínica em Odontologia. Revista Fluminense de Odontologia. Ano XXIII. N47. Janeiro-Junho 2017.

COLHADO, O.C.G.; BOEING, M.; ORTEGA, L.B. Toxina botulínica no tratamento da dor. Revista Brasileira de Anestesiologia. v.59, n.3, p. 366-38, 2009.

CÔRTE-REAL DE CARVALHO, R.; SHIMAOKA, A.M.; ANDRADE, A.P. O uso da toxina botulínica na Odontologia. Disponível em: [http://cfo.org. br/wpcontent/uploads/2011/05/toxina-botulinica.pdf]. Acesso em: 15/04/2020. 
CRITCHFIELD, J. Considering the immune response to botulinum toxin. Clin $\mathbf{J}$ Pain. 2002; v. 18, p: S133-S141.

DA ESCÓSSIA, N.B.M.; FERRAZ L.K.; JÚNIOR, J.C. Utilizaçãode toxina botulínica do tipo A para minimizar o sorriso gen-gival: relato de três casos clínicos. Rev Clín Ortod Dental Press, 2014. v.13, ed. 4, p.68-73

DAYAKAR, M.M.; GUPTA, S.; SHIVANANDA, H. Lip repositioning: An alternative cosmetic treatment for gummy smile. J Indian Soc Periodontol, 2014. v. 18, p. 52023.

DELPACHITRA, S.N.; SKLAVOS, A.W.; DASTARAN, M. Usos clínicos da toxina botulínica A na modificação estética do sorriso. Br Dent J, 2018. v.225, p. 502-506.

DINKER S, ANITHA A, SORAKE A. KUMAR K. Management of gummy smile with. Botulinum Toxin Type-A: A case report. J Int Oral Health 2014; v.6, p. 111-115.

DRESSLER D, ELEOPRA R. Clinical use of non-a botulinum toxins: Botulinum toxin type b. Neurotox Res. 2006, v.9, p.121-125.

DUTRA MB, et al. Influência da exposição gengival na estética do sorriso. Dental Press J Orthod, 2011. v. 16, p. 5111-5118.

EMARA AS, FARAMAWEY MI, HASSAAN MA, HAKAM MM. Botu-linum toxin injection for management of temporomandibular joint clicking. Int $\mathbf{J}$ Oral Maxillofac Surg 2013;42(6):759-64.24.

FAVRE-GUILMARD, C.; CHABRIER, P. E.; KALINICHEV, M. Bilateral analgesic effects of abobotulinumtoxinA (Dysport O) following unilateral administration in the rat. Eur J Pain, v. 21, n.1, p. 927-937, 2017.

GRACCO A, TRACEY S. Botox and the gummy smile. Prog Orthod. 2010;11(1):7682.

HUANG, W.; FOSTER, J.A.; ROGACHEFSKY, A.S. Pharmacology of botulinum toxin. Journal of the American Academy of Dermatology, 2000. v. 43, n. 2, p. 249-259.

INDRA A.S.; BISWAS, P.P.; VINEET, V.T.; YESHASWINI, T. Botox as an adjunct to orthognathic surgery for a case of severe vertical maxillary excesso. J Maxillofac Oral Surg, 2011. v.10, p. 266-270.

ISHIDA, L.H. Estudo das alterações do sorriso em pacientes submetidas a alongamento do lábio superior associado à miotomia do músculo levantador do lábio superior. Tese [Doutoramento em Ciências] - Faculdade de Medicina da Universidade de São Paulo, São Paulo, 2012.

JANANNI. M.; SIVARAMAKRISHNAN, M.; LIBBY, T.J. Surgical correction of excessive gingival display in class I vertical maxillary excess: Mucosal strip technique. J Nat Sci Biol Med, 2014. v. 5, n. 2, p. 494-8.

JANKOVIC J. Botulinum toxin in clinical practice. Neurol Neu-rosurg Psychiatry 
JASPERS G. W. C, PIJPE J, JANSMA J. The use of botulinum toxin type A in cosmetic facial procedures. Int J Oral Maxillofac Surg. 2011; v. 40, n.2, p. 127-33.

KANE, M.; SATTLER, G. Guia ilustrado para infiltrações estéticas com toxina botulínica. Dilivros: Rio de Janeiro, 2013. 182p.

KESSLER, K.R.; SKUTTA, M.; BENECKE, R. Long-term treatment of cervical dystonia with botulinum toxin a: Efficacy, safety, and antibody frequency. German dystonia study group. J Neurol. 1999; v.246, p. 265-274.

KUHN-DALL'MAGRO, A.; CALZA, S. C.; LAUXEN, J.; SANTOS, R.; VALCANAIA, T. C.; DALL'MAGRO, E. Tratamento do sorriso gengival com toxina botulínica tipo A: relato de caso. Revista da Faculdade de Odontologia, 2015. v. 20, p. 81-87.

MAJZOUB, Z.A.K.; ROMANOS, A.; CORDIOLI, G. Crown lengthening procedures: A literature Review. Semin Orthod, 2014. v. 20, p.188-207.

MANTOVANI, M.B.; SOUZA, E.C.; MARSON, F.C.; CORRÊA, G.O. PROGIANTE, P.S.; SILVA, C.O. Use of modified lip repositioning technique associated with esthetic crown lengthening for treatment of excessive gingival display: A case report of multiple etiologies. J Indian Soc Periodontol, 2016. v.20, p. 82-87.

MARCIANO, A.; AGUIAR, U.; VIEIRA, P. G. M.; MAGALHÃES, S. R. Toxina Botulínica e sua aplicação na odontologia. Revista de Iniciação Científica da Universidade Vale do Rio Verde, Três Corações, v. 4, n. 1, 2014, p. 65-75.

MARSON F.C. et al.Análise da Interrelação entre estética periodon-tal e dentária. Revi Dental Press Estét 2012; v. 9, n. 2, p. 58-68.

MARSON, F.C.; PILOTO, R.L.; ROCHA, O.O.; LOLLI, L.F.; PROGIANTE, P.S.; SILVA, C.O. Percepção da atratividade do sorriso. RevUNINGÁ Review, 2014; v. 20, p. 26-9.

MAZZUCO, R.; HEXSEL, D. Gummy smile and botulinum toxin: A new approach based on the gingival exposure area. Journal of the American Academy of Dermatology, 2010. v. 63, n. 6, p. 1042-1051.

MOREIRA, D.C.; POSSIDÔNIO, F.S.; SOUZA, F.S.; KINOSHITA, A.M.O.; SILVEIRA, E.M.V. Application of botulinum toxin type A in gymmy smile: case report. Rev Gaúch Odont, 2019. v.67, p. 1-5.

MOSTAFA, D. A successful management of sever gummy smile using gingivectomy and botulinum toxin injection: A case report. International Journal of Surgery Case Reports, 2018. v.42, p. 169-174.

MÜNCHAU, A.; BHATIA, K.P. Uses of botulinum toxin injection in medicine today. BMJ. 2000; v.320, n. 7228, p. 161-165.

MUTHUKUMAR, S.; NATARAJAN, S.; SAMPATHKUMAR, J. Lip repositioning surgery for correction of excessive gingival display. J Pharm Bioallied Sci, 2015. v.7, p. 794- 
96.

NAUMANN M, ALBANESE A, HEINEN F, MOLENAERS G, Relja M. Safety and efficacy of botulinum toxin type a following long-term use. Eur J Neurol. 2006; v. 13, p. 35-40.

NIAMTU, J. Botox injections for gummy smiles. Am J Orthod Dentofacial Orthop. 2008; v. 133, n. 6, p. 782-3.

NIGAM, P.K.; NIGAM A. Botulinum Toxin. Indian J Dematol 2010; v.55, p. 8-14.

OLIVEIRA, C.C.A.; FERNANDES, E.C.; MEDEIROS, K.D.O.; MAIA, M.C.B.; SEABRA, E.J.G.; SANTO, P.C. Toxina botulínica: contexto histórico, molecular e de aplicação prática na área da saúde. Revista Brasileira de Educação e Saúde, 2020. v. 10, n. 2, p. 1-10.

PARK, K-S.; LEE, C-H.; LEE, J-W. Use of a Botulinum Toxin A in Dentistry and Oral and Maxillofacial Surgery. J Dent Anesth Pain Med, 2016. v, 16, n.3, p. 151-157.

PEDRON, I.G. Aplicação da toxina botulínica associada à cirurgia gengival ressectiva no manejo do sorriso gengiva. RFO, Passo Fundo, v. 20, n. 2, p. 243-247, 2015.

PEDRON, I.G. Associação terapêutica entre cirurgia gengival ressectiva e aplicação de toxina botulínica no sorriso gengival em paciente ortodôntico. Ortodontia SPO, 2014a; v.47, n.3, p.245-9.

PEDRON, I.G. Utilização da toxina botulínica tipo $A$ associada à cirurgia gengival ressectiva: relato de caso. Brazilian Journal of Periodontology, 2014b. v. 24, n. 3, p.35-39.

PERÃO, P.C.B.G.; BARBOSA, F.K.Toxina Botulínica A. Revista UNILUS Ensino e Pesquisa, v.12, n. 27, 2015.

POLO, M. Botulinum toxin type A (Botox) for the neuromuscular correction of excessive gingival display on smiling (gummy smile). Am J Orthod and Dentofac Orthop 2008 Feb; v. 133, n. 2, p. 195-203.

POLO, M. Botulinum toxin type A in the treatment of excessive gingival display. Am $\mathbf{J}$ Orthod Dentofacial Orthop. 2005; v.127, n.2, p.214-8.

ROCHA, A.C.; UTUMI, E.R.; PEDRON, I.G. Sialorreia. In: Caldas Neto S, Mello Jr JF, Martins RHG, Costa SS. Tratado de Otorrinolaringologia. São Paulo: Ed. Roca, 2011, 149-53

ROSSI, R.; BRUNELLI, G.; PIRAS, V.; PILLONI, A. Altered Passive Eruption and Familial Trait: A Preliminary Investigation. Int J Dent, 2014. v. 1, p. 1-5.

SABRI, R. The Eight Components of a Balanced Smile. JCO, 2005. v.39, n. 3, p.155167.

SANDLER J, ALSAYER F, DAVIES J. Botox: a possible new treat-ment for gummy 
smile. Virtual Journal of Orthodontics (se-rial online), 2007;7(4):30-34. Feb. 20, 2007. Disponível em: <http://www.vjo.it/wp-content/uploads/2010/09/botox.pdf>. Acesso em: 16/05/2020.

SARVER, D.M.; ACKERMAN, M.B. Dynamic smile visualization and quantification: Part 2. Smile analysis and treatment strategies. Orthod Dentofacial Orthop. 2003; v.124, p. 116-27.

SATRIYASA, B.K. Botulinum toxin (Botox) A for reducing the appearance of facial wrinkles: a literature review of clinical use and pharmacological aspect. Clin Cosmet Investig Dermatol. 2019. v. 10, n.12, p. 223-228. .

SCHANTZ, E.J.; JOHNSON, E.A. Properties and Use of Botulinum Toxin and Other Microbial Neurotoxins in Medicine. Microbiological reviews, 1992. v. 56, p. 80-99.

SEIXAS, M.R.; COSTA-PINTO, R.A.; ARAÚJO, T.M. Checklist of esthetic features to consider in diagnosing and treating excessive gingival display (gummy smile). Dental Press J Orthod, 2011. v. 16, n. 2, p. 131-57.

SEIXAS, M.R.; COSTA-PINTO, R.A.; DE ARAÚJO, T.M. Checklist of esthetic features to consider in diagnosing and treating excessive gingival display (gummy smile) Dental Press J Orthod. 2011; v.16, p.131-57.

SENISE, I.R.; MARSON, F.C.; PROGIANTE, P.S.; SILVA, C.O. O uso de toxina botulinica como alternativa para o tratamento do sorriso gengival causado pela hiperatividade do lábio superior. Revista Uningá Review, 2015. v.23, n.3, p. 104-110.

SILVA, J.F.N. A aplicação da Toxina Botulínica e suas complicações. Revisão Bibliográfica. Porto: Instituto de Ciências Biomédicas de Abel Salazar da Universidade do Porto, 2009.

SPOSITO, M.M.M. Toxina botulínica tipo A - propriedades farmacológicas e uso clínico. Acta Fisiátr. 2004 v.1, p.7-44.

SRIVASTAVA, S.; KHARBANDA, S.; PAL, U.; SHAH, V. Applications of botulinum toxin in dentistry: A comprehensive review. Natl J Maxillofac Surg. 2015, v.6, n. 2, p.152-9.

TIGHE, A.P.; SCHIAVO, G. Botulinum neurotoxins: Mechanism of action. New York: Elsevier, v.1, 2012.

VAN DER GELD, P. OOSTERVELD, P. SCHOLS, J. KUIJPERS-JAGTMAN, A.M. Smile line assessment comparing quantitative measurement and visual estimation. Am J Orthod Dentofacial Orthop, 2011. v. 139, n.2, p. 174-180.

WHEELER, A.; SMITH, H. Botulinum toxins: Mechanisms of action, antinociception and clinical applications. New York: Elsevier, v.1, 2013.

ZAGUI, R.M.N.; MATAYOSHI, S.; MOURA, F.C.; Efeitos adversos associados à aplicação de toxina botulínica na face: revisão sistemática com metaanálise. Arq Bras Oftalmol. 2008; v.71, n.6, p.894-901. 